Cahiers $d u$ MONDE RUSSE

\section{Cahiers du monde russe}

Russie - Empire russe - Union soviétique et États indépendants

$51 / 4 \mid 2010$

Sciences humaines et sociales en Russie à l'Âge d'argent

\title{
David C. Engerman, Know your Enemy
}

\section{Andreï Kozovoï}

\section{(2) OpenEdition}

1 Journals

Édition électronique

URL : https://journals.openedition.org/monderusse/7419

DOI : 10.4000/monderusse.7419

ISSN : $1777-5388$

Éditeur

Éditions de l'EHESS

Édition imprimée

Date de publication : 25 novembre 2010

Pagination : 766-771

ISBN : 978-2-7132-2316-7

ISSN : $1252-6576$

Référence électronique

Andreï Kozovoï, « David C. Engerman, Know your Enemy », Cahiers du monde russe [En ligne], 51/4 I 2010, mis en ligne le 09 décembre 2011, consulté le 03 septembre 2022. URL : http://

journals.openedition.org/monderusse/7419; DOI : https://doi.org/10.4000/monderusse.7419

Ce document a été généré automatiquement le 3 septembre 2022

Tous droits réservés 


\title{
David C. Engerman, Know your Enemy
}

\author{
Andreï Kozovoï
}

\section{RÉFÉRENCE}

David C. ENGERMAn, Know your Enemy. The Rise and Fall of America's Soviet

Experts. Oxford - New York : Oxford University Press, 2009, 459 p.

David Engerman est l'auteur de Modernization from the other Shore: American Intellectuals and the Romance of Russian Development (Harvard University Press, 2004), qui avait retracé le regard des États-Unis sur la Russie de la fin du XIX ${ }^{\mathrm{e}}$ siècle à 1939. Know your Enemy constitue une suite logique de cet ouvrage, puisqu'y est étudiée la vision de la Russie par les universitaires américains pendant la guerre froide, ainsi que l'impact du contexte de guerre froide sur l'historiographie américaine de la Russie. L'auteur fait partie d'une nouvelle génération d'historiens qui étudient ce phénomène « vu d'en bas ».

La propagande américaine de cette période a reçu l'attention de plusieurs spécialistes, notamment Kenneth Osgood, James Schwoch, Laura Belmonte et Nicholas Cull. Ce renouveau d'intérêt pour la guerre froide s'explique notamment par un contexte de séduction du monde arabe et de lutte contre le terrorisme islamiste.

Le livre est divisé en trois parties et comprend douze chapitres. La première partie («Un champ en formation ") décrit l'émergence et l'âge d'or des études russes et soviétiques américaines pendant la Seconde Guerre mondiale, jusqu'au milieu des années 1960. Les États-Unis partent de loin : avant la guerre, le champ est quasi vierge. Le conflit jouera le rôle de catalyseur. Après l'invasion de l'URSS en 1941, les décideurs américains ont besoin de mieux connaître le nouvel allié. Parmi les spécialistes qui ont le vent en poupe, on trouve Philip Mosely, issu de Harvard ; grâce au soutien de la fondation Rockefeller, il fonde un centre d'études russes à Cornell (Ithaca), promu à un bel avenir. Les militaires et les hommes du renseignement (l'OSS, ancêtre de la CIA) ont également besoin de spécialistes qui connaissent le russe : l'US Navy finance un programme d'apprentissage 
intense de la langue; parmi ses recrues figure un certain Martin Malia. Dans les formateurs du programme se trouve Adam Ulam, l'un des pères fondateurs de la soviétologie. Globalement, les hommes du gouvernement, les militaires et l'OSS, intéressés par des expertises interdisciplinaires (histoire, économie, sciences politiques, sociologie, linguistique, etc.), contribuent à faire émerger les area studies, centrées sur une région géographique particulière.

4 La fin du conflit et le début de la guerre froide ne font que renforcer cette tendance. Si d'autres pôles que Cornell se renforcent alors, à l'image de Stanford, réputée pour sa Hoover Institution on War, Revolution and Peace - centre d'archives russes sans équivalent dans le monde -, les principaux bienfaiteurs que sont les fondations Rockefeller et Carnegie privilégient le centre de Mosely qui devient après 1947 le Russian Institute. Avec son premier directeur, Geroid Robinson, Columbia pose les bases de l'infrastructure des Russian studies et forme de nombreux russisants, d'abord par un apprentissage de la langue - la recherche venant en second.

5 Après Cornell, l'université d'Harvard contribue à théoriser les Russian studies. Des hommes comme Talcott Parsons se posent en experts dont la mission consiste à aider l'exécutif américain à éviter une troisième guerre mondiale, nucléaire celle-ci. Parsons propose d'appliquer le behaviorisme, branche de la psychologie qui pose le primat de l'observation des comportements, pour étudier l'Union soviétique. Le champ d'application en sera les fameux « débats atomiques » à l'ONU. L'entreprise aboutit à la création du Russian Research Center (RRC) de Harvard. Le RRC devient rapidement un think tank pour les militaires, le département d'État et la CIA. L'un des projets les plus importants du RRC, commandé par l'US Air Force, porte sur des interviews menées dans les camps de réfugiés soviétiques en Allemagne de l'Ouest, sources de nombreuses monographies à venir. Le rapport final de l'enquête, "How the Soviet system works » (1956), met en évidence les "stratégies d'adaptation» de la population au régime et montre qu'à l'inverse d'une idée reçue, seul un groupe de la population soviétique apparaît vraiment hostile au régime : la paysannerie.

La décennie qui suit voit un développement extrêmement rapide. À cela plusieurs facteurs, internes et externes : relance de la recherche spatiale et scientifique américaine par suite du lancement du Sputnik par les Soviétiques en 1957; promulgation du National Defense Education Act (1958); accord Lacy-Zaroubine (1958) facilitant les échanges entre étudiants et chercheurs des deux pays. Est alors créée l'American Association for the Advancement of Slavic Studies (AAASS), ainsi que le Center for Slavic Studies à Berkeley. Dans ce contexte, l'intérêt pour la Russie dans le grand public ne cesse d'augmenter. En revanche, dès la première partie des années 1960, certains observent déjà des signes d'une crise des Russian studies (baisse de l'apprentissage de la langue russe ; recentrement sur la politique étrangère ; spécialisation excessive).

Dans une deuxième partie thématique, intitulée "Croissance et essaimage ", Engerman étudie successivement le cas des études économiques, littéraires, sociologiques, historiques et politiques de la Russie. Pour les spécialistes de littérature russe, les années 1950 et 1960 sont fastes. Les échanges offrent des possibilités inespérées pour étudier le russe. Ernest Simmons (Columbia) est l'un des slavisants les plus actifs de sa génération. Ses étudiants prennent la relève: en étudiant la réception de la littérature américaine en URSS (1962), Deming Brown devient un pionnier en histoire culturelle de la littérature. Mais, dans l'ensemble, les slavisants se montrent peu présents dans le débat public et sont singulièrement silencieux dès lors qu'il s'agit de condamner les 
manifestations du totalitarisme dans le domaine littéraire. L'exception est Gleb Struve, fils de l'émigré russe Pierre Struve, membre du parti constitutionnel-démocrate ayant fait partie du Gouvernement Provisoire. Le jeune Struve fait découvrir aux Américains la littérature interdite de Pasternak, Ahmatova, Mandel'štam et Gumilev; grâce à Martin Malia, historien de Berkeley qui passe l'année 1962 à Moscou, de nombreuses œuvres réussissent à franchir le rideau de fer. Mais, dans l'ensemble, c'est à la littérature classique que profite cet engouement, entre autres à la (re)découverte de Dostoevskij.

Les études d'histoire de la Russie sont, quant à elles, une affaire d'émigrés souvent empreints de la même culture K-D : l'auteur étudie les destins de George Vernadsky à Yale, Michael Karpovich et Alexander Gerschenkron à Harvard, auxquels il faut ajouter Isaiah Berlin d'Oxford, qui vient souvent aux États-Unis: tous ont une influence considérable sur les jeunes chercheurs. Karpovich a ainsi formé plusieurs générations d'historiens (dont Martin Malia et Richard Pipes) et permis la diffusion de revues phares comme la Russian Review. Mais les historiens de l'URSS rencontrent les mêmes difficultés que les autres spécialistes de la Russie dans l'accès aux archives.

9 La sociologie apparaît comme le parent pauvre des Russian studies. Il en va tout autrement pour les études politiques. Engerman décrit bien l'évolution de la notion de totalitarisme aux États-Unis, avec comme représentant célèbre Merle Fainsod (Harvard), dont l'ouvrage How Russia is ruled (1953), devenu un classique, présente le bolchevisme présent à l'état embryonnaire dans l'autocratie. La conclusion est pessimiste: «Le régime totalitaire ne se dépouille pas de ses traits d'État policier; il meurt quand le pouvoir lui est arraché des mains». Fainsod est le premier à étudier «les archives de Smolensk », soit 200000 pages de documents capturés par les Allemands en 1941 et passés aux Américains (Smolensk under the Soviet rule, 1958). En 1963, lorsque How Russia is ruled est réédité, la tonalité négative est revue à la baisse. Engerman conclut que lorsqu'une nouvelle génération d'historiens de l'URSS, les « révisionnistes ", fait du totalitarisme leur ennemi numéro un à la fin des années 1960, le terme est déjà passé de mode dans le monde universitaire (quoiqu'il reste utilisé dans les médias) : aucune " école totalitaire " n'existe alors et, d'une certaine manière, les révisionnistes se battent contre un fantôme.

La troisième partie, "Crise, conflit et chute ", s'intéresse aux années 1968-1991. Un chapitre chronologique évoque d'abord la fin des années fastes ainsi que la disparition de trois figures phares de la soviétologie, Fainsod, Mosely et Llewellyn Thompson (ambassadeur américain en URSS). Les financements des décennies précédentes fondent : le contexte est en effet aux difficultés intérieures, liées à la guerre au Vietnam, puis au scandale du Watergate, ainsi qu'à la crise pétrolière de 1973. Les area studies en général, et les Russian studies en particulier, sont touchées de plein fouet. Par ailleurs, la politique de détente ne signifie pas que les chercheurs américains aient plus accès aux archives soviétiques qu'auparavant. Des voix s'élèvent pour critiquer la politique des échanges, vue comme stérile. Enfin, l'ère de la création de postes dans les Russian studies est révolue, d'où la multiplication de conflits au sein des disciplines.

Ceci concerne d'abord les spécialistes qualifiés par Engerman de «conservateurs ». De plus en plus en rupture avec leurs universités (trop coupées de la vie publique), leurs étudiants (contestataires) et la politique du gouvernement américain, ceux-ci cherchent à se faire entendre dans le débat public, notamment par le biais de revues comme Foreign Affairs. Certains, comme Zbigniew Brzezinski, font des choix de carrière orientés en fonction de leurs ambitions politiques. Outre le futur conseiller du président Carter pour la sécurité nationale, Richard Pipes, Adam Ulam et Robert Conquest apparaissent comme 
les principaux représentants, aux vues parfois divergentes, de ce courant anti-détente. Pipes se fait connaître avec son livre The Formation of the Soviet Union, 1917-1923 (1954), où il évoque le potentiel déstabilisateur des nationalités non russes. Tout en enseignant à Harvard, il participe à la « Team B » en 1976, think tank destiné à évaluer les estimations de la CIA, puis, sous Reagan, devient expert de l'URSS au NSC.

La plupart des soviétologues sont cependant d'accord avec le cours de la détente impulsé par Nixon, à l'image d'un Stephen Cohen. Mais avec «l'ère des contestations » de la fin des années 1960 dans le domaine public s'ouvre «l'ère des révisions » dans le domaine scientifique. La guerre froide fait l'objet d'une réécriture, les États-Unis sont accusés de porter une lourde responsabilité dans son déclenchement. Dans le domaine historique, l'histoire soviétique est également révisée : ainsi, Cohen réhabilite Buharin, Moshe Lewin et Sheila Fitzpatrick réduisent l'image noire de Stalin en évoquant une responsabilité collective et en déplaçant l'accent de la terreur à la révolution sociale.

Dans le dernier chapitre, David Engerman évoque les conséquences de la perestroïka et de la fin de l'URSS sur les Russian studies. L'idée centrale de l'auteur est que l'ensemble des soviétologues se révèle incapable d'expliquer l'avènement de Gorbačev, ses réformes, et encore moins d'anticiper la fin du régime, tout en étant conscients que l'économie soviétique se trouve dans une situation catastrophique. Des spécialistes aussi opposés idéologiquement que Stephen Cohen et Richard Pipes abordent cette période avec des œillères qui les empêchent de saisir la nouveauté du tournant historique. L'opposition se cristallise sur la sincérité de la démarche de Gorbačev et le cours à suivre pour la politique américaine. La disparition de l'URSS en 1991 entraîne un nouveau débat au sein des disciplines des Russian studies qui ont participé aux analyses à chaud des événements. Pour Engerman, la faute n'est cependant pas à un quelconque «vice » structurel de la soviétologie, mais au contexte de crise et de débats peu productifs que vit la discipline depuis les années 1970.

14 Cette monographie vient moins combler une lacune historiographique que rassembler de nombreuses pièces d'un puzzle étudié depuis plusieurs années, celui de l'histoire de la Russie telle qu'elle s'est écrite pendant la guerre froide; c'est donc plus un bilan qu'un véritable tournant. L'auteur brosse une histoire très dense, qui fourmille de détails, une véritable encyclopédie des spécialistes américains de la Russie, de leurs idées et théories, ainsi qu'un historique des principales institutions. Basé sur des sources essentiellement américaines - d'abord celles d'Harvard, l'ouvrage s'appuie aussi sur des sources russes: outre des périodiques d'époque, l'auteur affirme avoir consulté le fonds 5 du RGANI, et les fonds 8131 du GARF (dont on ne trouve pas trace des notes). Il a bénéficié également des apports de l'histoire orale avec, entre autres, une interview de Martin Malia.

Ce travail comporte cependant un certain nombre de carences. Des simplifications, dues au choix d'un sujet trop vaste ou à une approche trop idéologisée, sont à regretter dans la présentation aussi succincte que caricaturale des années 1950-1954, où le sénateur McCarthy est qualifié de « Grand Inquisiteur » (p. 42), alors que la tendance actuelle est à une réévaluation prudente de la période et du personnage. On note aussi l'usage indifférencié d'expressions telles que "soviétologie», "Russian studies» et "Soviet studies "; celles d'area studies, "academic experts ", "scientists ", "scholars", "intelligence analysts" ne sont jamais expliquées clairement. Ce qui entraîne un problème d'interprétation de l'ensemble: quoi de commun entre un spécialiste de Puškin et un politologue de l'URSS, outre le fait qu'ils étudient une entité géographique et culturelle commune? Leurs intérêts institutionnels ne peuvent être confondus. Vu l'absence de 
délimitation des acteurs étudiés, le lecteur se demande pourquoi l'auteur n'a pas jugé bon de traiter l'impact de la guerre froide sur le travail d'universitaires comme Henry Kissinger (Harvard) ou John Lewis Gaddis (Yale) : n'étant pas des soviétologues au sens premier du terme, ceux-ci ont cependant tous deux abordé l'URSS dans leurs travaux et joué un rôle important dans la vie intellectuelle américaine, sans parler de l'élaboration de la politique étrangère. Enfin, on peut reprocher à l'auteur d'avoir étudié les « experts de l'URSS » en vase clos, sans prise avec le réel. Refuser d'emblée l'hypothèse que les " experts» ne forment pas seulement l'opinion publique, mais qu'ils sont aussi formés par elle, conduit à une impasse.

Enfin, les conclusions de l'auteur méritent discussion. L'épilogue du ch. XII, évoquant l'échec des soviétologues à " prédire » la fin de l'URSS, semble spécieux. À quelques rares exceptions près, la fin de l'URSS ne faisait pas partie d'un horizon d'attente partagé. Le sentiment que l'URSS allait continuer à se décomposer de l'intérieur encore de longues années n'était pas l'apanage d'un petit groupe de chercheurs, mais correspondait au contraire à l'opinion d'une bonne partie de la population soviétique. Dès lors, il convient de relativiser la notion de «crise » et de " chute » proposée par les experts américains. Et de noter que le principal mérite de ce livre est d'inciter à beaucoup d'humilité en matière de prédictions. Au final, toute histoire, y compris russe, réserve son lot de surprises et de coups de théâtre. Reste à savoir comment appliquer « la leçon de 1991 » à notre regard sur la nouvelle Russie de Putin-Medvedev et son avenir de démocratie incertaine. 